\title{
I wanna draw like you: Inter- and intra-individual differences in orang-utan drawings
}

\author{
Marie Pelé ${ }^{1}$, Gwendoline Thomas², Alaïs Liénard ${ }^{2}$, Nagi Eguchi' ${ }^{3}$, Masaki Shimada ${ }^{3}$, Cédric Sueur ${ }^{4,5}$ \\ 1: Anthropo-Lab, ETHICS EA7446, Lille Catholic University, Lille, France \\ 2: Université Sorbonne Paris Nord - UFR LLSHS, Paris, France \\ 3: Department of Animal Sciences, Teikyo, University of Science, Uenohara, Yamanashi, Japan \\ 4 : Université de Strasbourg, CNRS, IPHC UMR 7178, Strasbourg, France \\ 5: Institut Universitaire de France, Paris, France \\ * Corresponding author: Marie Pelé, Lille Catholic University, marie.pele@univ-catholille.fr
}

Simple Summary: Drawing is increasingly proposed as an enrichment activity for captive primates in zoological parks and research institutes. The monkeys and apes are free to use the material at their disposal, and are not constrained or conditioned to show this behaviour. This provides a perfect opportunity to collect drawings by non-human primates and allows comparative studies between hominids. This study is based on 1,433 drawings recovered from five orang-utans (Pongo pygmaeus) at Tama Zoological Park in Japan, where caretakers regularly proposed drawing activity to the apes. Analyses showed that individuals differ in their drawing style, especially in the colors used, the space they filled and the shaped they draw. One individual, Molly, did more complex drawings than other individuals and draw differently according to the seasons and her age. This study is the first to reveal such individual differences and can give some cues about the emergence of drawings in human beings.

\begin{abstract}
This study analyses 749 drawings of five female Bornean orang-utans (Pongo pygmaeus) at Tama Zoological Park in Japan. We searched for differences between individuals but also tried to identify possible temporal changes among the drawings of one individual, Molly, who drew almost 1,300 drawings from 2006 to 2016. An analysis of the drawings was carried out after collecting quantitative and qualitative variables. Our findings reveal evidence of differences in the drawing style of the five individuals as well as creative changes in Molly's drawing style throughout her lifetime. Individuals differed in terms of the colours used, the space they filled but also the shapes (fan patterns, circles or loops) they drew. Molly drew less and less as she grew older, and we found a significant difference between drawings produced in winter, when orang-utans were kept inside and had less activity, and those produced during other seasons. Our results suggest that the drawing behaviour of these five orang-utans is not random and that differences among individuals might reflect differences of styles, states of mind but also motivation to draw.
\end{abstract}

Keywords: Primate cognition; scribbles; evolutive anthropology; art; aesthetics

\section{Introduction}

Drawing behaviour has been studied in non-human primate species including chimpanzees (Pan troglodytes), gorillas (Gorilla gorilla), orang-utans (Pongo pygmaeus), capuchin monkeys (Cebus apella) and rhesus macaques (Macaca mulatta) (for a review, see [1]). Nowadays, drawing is increasingly proposed as an enrichment activity for captive primates in zoological parks and research institutes. The monkeys and apes are free to use the material at their disposal, and are not constrained or conditioned to show this behaviour. This provides a perfect opportunity to collect drawings by non-human primates and allows comparative studies between hominids [2]. 
This study is based on 1,433 drawings recovered from five orang-utans (Pongo pygmaeus) at Tama Zoological Park in Japan, where caretakers regularly proposed drawing activity to the apes. Orang-utans (Pongo sp.) are phylogenetically close to humans, with $97 \%$ of common genetic heritage [3]. This makes them ideal candidates to help us understand the origins of drawing behaviour. They can develop highly sophisticated cognitive abilities [4,5] and complex tool use [6-8], that are both at least comparable to those observed in chimpanzees. They are also well known for their curiosity [9]. The wrists of orangutans are more flexible than those of chimpanzees, making it easier for them to draw as they can bend their hands backwards [10]. In our drawings database, the daily number of drawings varies significantly between individuals -despite same opportunities to draw, showing that they had different levels of motivation for this activity. This observation leads us to a first question: Do orang-utans show differences in their drawing/marking behaviour? More specifically, it is interesting to note that one female, called Molly, drew almost 1,300 drawings in her last five years of life. She had the opportunity to draw regularly from her 54th birthday onwards (in 2006, at Tama Zoo). Moreover, the colours and page filling in Molly's drawings might be affected by aspects of her daily life such as the identity of her caretaker and events in her environment [11]. This leads us to a second question: Is there any temporal evolution (due to environmental changes and/or Molly's own development) in her drawing behaviour?

Previous studies on captive orang-utans and other primates showed that they will continue to draw even in the absence of rewards [11-14]. These findings are consistent with the Gestalt principle found in young children, which links the scribbling activity to a discovery of motor play activity [15]. Like in humans, spontaneous drawings indicate an intrinsic interest in exploratory and manipulative play for captive non-human primates $[11,15]$. Moreover, when tracks have already been drawn on the paper, further scribblings are added, suggesting that visible tracks have some kind of reinforcing value [13]. Schiller [16] worked with an 18-year-old female chimpanzee called Alpha, who showed a keen interest in drawing. In order to study figure formation and position, different stimuli (squares or scribbles) were drawn on paper by researchers. In terms of figure production, the results showed that Alpha mainly used two types of strokes: short dashes, and almost parallel broad zigzag strokes (also referred to as the fan pattern). Findings for the placement of figures reveal that when a single figure was positioned off-centre, Alpha drew in the largest open space, producing what Schiller calls a 'sort of balance between her markings and the presented figure' (p. 104). A similar experiment by Morris [17] with a one-year-old male chimpanzee named Congo showed similar results: three-quarters of the 40 free drawings on blank sheets of paper showed marks on spaces that had previously been empty, and half of the drawings featured marks that were concentrated at the centre of the paper. Although these results are only descriptive (like those found for Alpha), they do seem to support Schiller's findings concerning the strong tendency to mark a central figure and to position marks in the blank space opposite an offset figure, as well as the inclination to simply enjoy scribbling. Smith [18] was the first to use quantitative methods in order to analyse chimpanzee drawings. All his results were consistent with the previously cited findings. Later, Boysen et al. [12] continued the stimulus-drawing test in chimpanzees by presenting 18 different figures to three chimpanzees. Like Smith (1973), Boysen attained a point where the presence of any stimulus figure on the page elicited more centralised markings than in cases where the sheet of paper was blank. The notion of the centre of a page therefore seems to be an important point to take into consideration when studying the emergence of drawing capacities.

Kinematic aspects could be seen as precursors of a graphic representation [19]. Yet drawings by non-human primates include different types of marks, such as the straight lines, curves, loops or hook-like strokes observed in drawings by chimpanzees [13]. Although chimpanzees only develop the skill to use a mark-making instrument at the age 
of 20-23 months, these marks can be observed before the age of 13-23 months through the use of touch-screens. In a comparative study, a set of 396 pictures was collected, made up of 40 drawings by chimpanzees, 153 by gorillas, 146 by orang-utans, and 57 non-figurative drawings by children up to and including the age of four [20]. Zeller [20] noticed that the main features of orang-utans' patterns were diagonals, arcs, and curvilinear designs; those of gorillas contain mainly arcs and open curving lines, and a very high proportion of dots. In contrast, chimpanzees' drawings were mainly characterised by the use of straight and jointed lines. Unlike Tanaka, Zeller noted that orang-utans were the only apes that can use a closed loop or circular pattern [20]. This is the most difficult pattern to produce, because it requires high levels of motor control. Another important point concerns the use of colours. Findings in children have shown that their choice of colours reflected their emotional state at that time [21], and it seems that this may be the case in individuals of other species, as with the case of the orang-utan called Molly [11]. These results support the hypothesis that there is a choice in the use of colour, the type of strokes and the use of space, and that drawings in great apes do not result from a totally random scribbles [2,20]. Despite their non-figurative nature, drawings produced by apes could therefore provide a great deal of meaningful information.

In this study, we analyse 790 drawings (selected from a total of 1,433 drawings) drawn by five orang-utans (Figure 1) and compare them at an inter-individual level. Indeed, a study of five chimpanzees by Morris [17] suggests that inter-individual differences exist in drawing and that personality may have an impact on the way marks are distributed. We analyse drawings at an individual level in order to assess temporal changes in this behaviour. We expect to find preferences pertaining to the use of colours, as well as a trend of centering drawings, a use of curved strokes and circles inside the drawings, and possibly a number of differences in marking behaviour between individuals. We also expect possible temporal changes in the drawings of one individual, Molly, who drew almost 1,300 drawings from 2006 to 2011. To our knowledge, this is the first study analysing such a large number of drawings by orang-utans, and indeed by non-human primates in general.
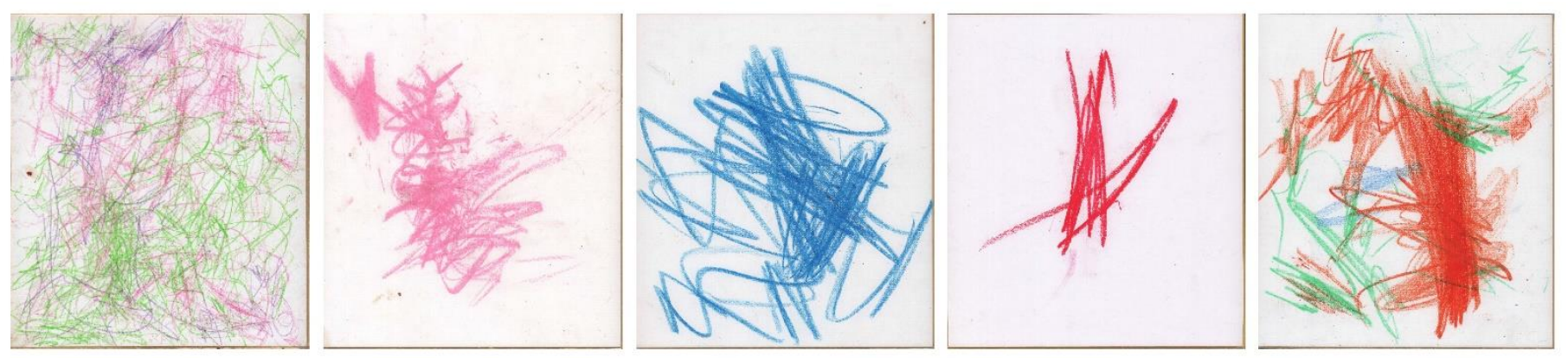

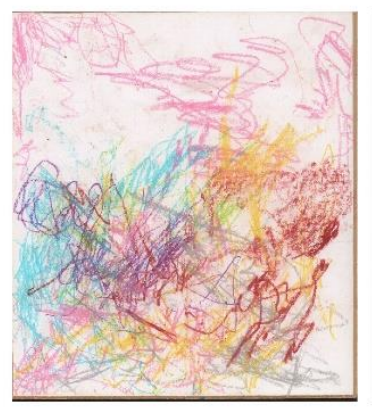

Molly

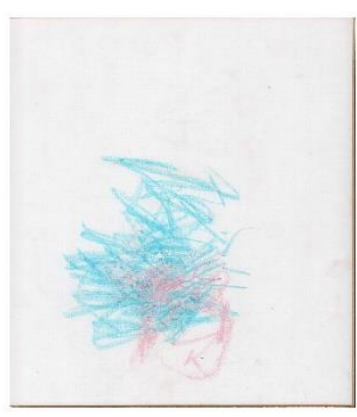

Gypsy

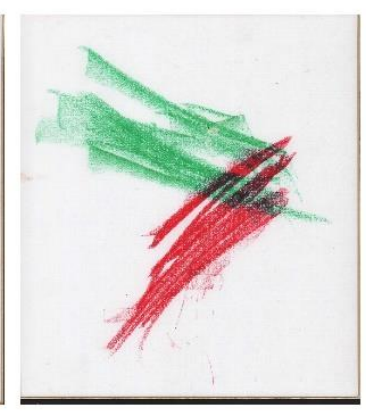

Julie

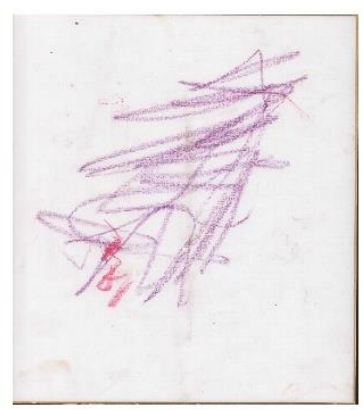

Kiki

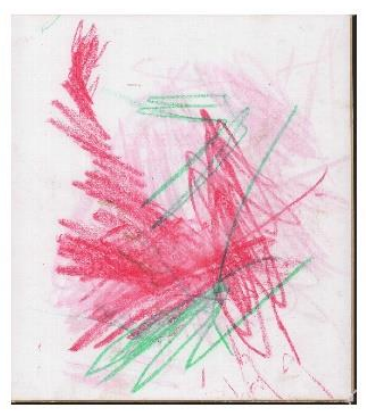

Yuki

Figure 1: Examples of two drawings for each of the five orang-utans. 


\section{Materials and Methods}

\subsection{Subjects and collection of drawings}

One thousand four hundred and thirty-three drawings by five female orang-utans at Tama Zoological Park in Tokyo were collected by the caretakers from 2006 to 2016 (Table 1). We analysed 749 drawings (26/26 drawings by Gypsy, 16/16 by Julie, 32/32 by Yuki, $60 / 60$ by Kiki, and 656/1299 by Molly). White, high-quality paperboard (272 x 242 $\mathrm{mm}$ ) and 16 different coloured crayons were proposed to the orang-utans as permanently available enrichment. Several paperboards were simultaneously provided to orang-utans to avoid competition, but no conflict was observed given the small number of drawings for four of the five individuals. The drawing activity, in the screened restroom, was not part of a research protocol implemented at the zoo but was for enrichment [22]. The drawings were recovered for analysis after the drawing activities, meaning that we had no control over the methodology. Individuals did not provide the same number of drawings each year, suggesting different motivations between apes and over time. We pseudo-randomly selected 656 drawings by Molly to obtain approximately equal numbers for each three-month period from 2006 to 2011 (about 30 drawings for each of the 19 periods; Molly died in 2011). The orang-utans were housed in social groups in outdoor and indoor enclosures with environmental enrichments. Food and water were supplied ad libitum.

Table 1. Information on individuals.

\begin{tabular}{|c|c|c|c|c|c|c|}
\hline Name & Date of Birth & Birthplace & $\begin{array}{c}\text { Number of } \\
\text { drawings }\end{array}$ & $\begin{array}{c}\text { Percentage of } \\
\text { drawings }\end{array}$ & Stay at Tama Zoo & Drawing period \\
\hline Molly & $01 / 01 / 1952$ & Wild & 1299 & $91 \%$ & $2005-2011$ & $2006-2011$ \\
\hline Gypsy & $01 / 01 / 1955$ & Wild & 26 & $2 \%$ & $1958-2017$ & $2007-2014$ \\
\hline Julie & $06 / 05 / 1965$ & Captivity & 16 & $1 \%$ & $2005-$ Today & $2009-2014$ \\
\hline Yuki & $01 / 01 / 1970$ & Wild & 32 & $2 \%$ & $2008-2015$ & $2008-2010$ \\
\hline Kiki & $21 / 10 / 2000$ & Wild & 60 & $4 \%$ & $2007-$ Today & $2010-2016$ \\
\hline
\end{tabular}

\subsection{Ethics note}

The Tama Zoological Park Ethics Board approved this non-invasive behavioural study, which complied with the Code of Ethics for Japanese Association of Zoos and Aquariums.

\subsection{Data collection}

To ensure that our observations were as accurate as possible, we used Gimp 2.10.22 software to apply a $10^{*} 10$ grid [15] to every drawing. Torn sheets were not analysed due to the risk of missing values. Two observers (GT and AL) made the measurements with a correlation of $0.964 \leq \mathrm{r} \leq 1$ for all variables (Figure S1 in Supplementary Material). A third observer (MP) then confirmed the qualitative variable measurements. Disagreements were resolved by consensus.

\section{- Quantitative variables}

We first looked for non-exclusive quantitative variables (Figure S2) as defined in previous studies [15]: (1) the coverage rate, defined as the number of cells containing one or more strokes out of the total number of cells, (2) the overlap rate, defined as the number of cells containing strokes of at least two different colours that overlap, divided by the coverage rate and multiplied by 100, (3) the solid colour rate, defined as the number of drawing grid cells that were covered at rates of $50 \%$ or more, divided by 100 , 
and (4) the distance to the centre, defined as the absolute distance between the centre of an ellipse surrounding the design and the centre of the grid. Three other indices were measured via Gimp 2.10.22 software, namely (5) the number of colours used, (6) the mean deviation of the colour spectrum and (7) the standard deviation of the colour spectrum.

\section{- Qualitative variables}

We also extracted data from every drawing (8) for the predominant colour used by the individual and the drawings shapes, as defined in previous studies [13,23]: (9) fan patterns, (10) circles, (11) triangles and (12) loops (Figure S3). These indices are not exclusive, as several can be found on the same drawing. A fan pattern is a stroke making at least three round trips of angles $\leq 45^{\circ}$. A loop is a curved stroke forming a single distinct angle where it intersects itself. A circle is a curved stroke intersecting in itself without distinct angles. A triangle is a flat loop with distinct angles.

\subsection{Statistical analysis}

We conducted two types of analyses: a comparative study between individuals and a longitudinal study of data for Molly. For both, we first checked for high correlations $(>80 \%)$ between our drawing variables using the chart.Correlation() function of the $\mathrm{R}$ package PerformanceAnalytics [24]. Our correlation chart revealed no strong correlation between our quantitative variables (Figure S4). A principal component analysis (PCA) was then carried out with the R package FactoMineR [25] in order to reduce our 12 variables and group them into various dimensions that were then further interpreted from a biological perspective. A PCA with Varimax rotation was also carried out [26] but did not explain more variance than classical PCA did. For the comparative study between individuals, the coordinates of each drawing in each dimension were used to compare individuals two by two during comparisons of means for each dimension with the functions kruskal_test() and pairwise.wilcox.test() from the R packages coin [27] and stats [28].

For the longitudinal study of data for Molly, we carried out another PCA. Here, we wanted to study the effect of both 3-month periods ( $N=19$ periods) corresponding to seasons ( $\mathrm{N}=4$ seasons) on Molly's drawing behaviour. We then applied a multifactorial linear model (LM) for each dimension of our PCA using the function $\operatorname{lm}()$ from the R package car [29]. The potential collinearity between our two predictor/predictive variables was tested by the calculation of the variance inflation factor VIF from the R package car. These diagnostics revealed a VIF of $<1.1$ for both predictors, indicating that there was no notable problem of collinearity. p-values for LM were calculated via Monte Carlo sampling with 10,000 permutations, using the function PermTest() of the R package pgirmess [30]. Permutation tests for LM were well adjusted for moderate sample size and did not require normal distribution of model residuals [31].

Pairwise post hoc comparisons for significant LMs were carried out with the function pairwisePermutationTest() from the $R$ package rcompanion [32]. $\alpha$ levels (0.05) were Benjamini-Hochberg corrected. Finally, we analysed the main colour used by orangutans with the function chisq.test() of the $\mathrm{R}$ package stats. We only reported only differences where $p<0.05$. All statistical analyses were done with $R$, version 4.0.3 [28].

\section{Results}

\subsection{Comparative analyses between individuals}

On average, the coverage rate of the drawings by the five orang-utans was $50 \pm 30 \%$, the overlap rate was $20 \pm 30 \%$ and the solid colour rate was $10 \pm 20 \%$. As regards the average number of colours used and shapes drawn, the five orang-utans used $3.0 \pm 1.8$ colours 
and drew $1.8 \pm 2.0$ fan patterns, $0.0 \pm 0.2$ circles, $0.2 \pm 0.6$ triangles, and $0.7 \pm 1.2$ loops per drawing. The colour spectrum had a mean of $0.8 \pm 0.1$ and a standard deviation of $0.1 \pm 0.1$. Finally, strokes were applied $33.7 \pm 25.9 \mathrm{~mm}$ from the centre, on average. Variations of each metric per individual are presented in Figure S5.

The three dimensions retained in the PCA had an eigenvalue above 1 and described $63.5 \%$ of the explained variance of the dataset. Each metric showed a higher loading in one dimension than in the two others (Table 2, Figure 2). Thus, the first dimension of the PCA (eigenvalue $=3.86$, variance $=35.2 \%$ ) was mainly explained by the filling variables: the recovery rate, the overlap rate, the solid colour rate, the number of fan patterns, the number of colours and the distance to the centre. Colour variables applied to the second dimension (eigenvalue $=1.92$, variance $=17.5 \%$ ), namely the standard deviation and the mean of the colour spectrum. Finally, geometrical shapes were associated with the third dimension (eigenvalue $=1.19$, variance $=10.8 \%$ ), i.e. the number of triangles, loops, and circles.

Table 2. Loadings of the metrics on the three PCA dimensions of our dataset (five orang-utans). Bold values indicate the dimension in which each variable is retained.

\begin{tabular}{|c|c|c|c|}
\hline & Dim.1 & Dim.2 & Dim.3 \\
\hline Coverage rate & $\mathbf{0 . 8 4}$ & 0.34 & -0.02 \\
\hline Overlap rate & $\mathbf{0 . 8 6}$ & 0.17 & -0.15 \\
\hline Number of colours & $\mathbf{0 . 7 2}$ & 0.32 & -0.26 \\
\hline Fan patterns & $\mathbf{0 . 7 6}$ & -0.12 & -0.05 \\
\hline Circles & 0.10 & 0.14 & $\mathbf{0 . 5 0}$ \\
\hline Triangles & 0.08 & 0.10 & $\mathbf{0 . 7 3}$ \\
\hline Loops & 0.33 & 0.31 & $\mathbf{0 . 5 0}$ \\
\hline Colour mean & -0.39 & $\mathbf{0 . 7 9}$ & -0.14 \\
\hline Std. deviation of & 0.38 & $\mathbf{- 0 . 8 2}$ & 0.15 \\
\hline colour mean & & -0.31 & -0.02 \\
\hline Distance to centre & $\mathbf{- 0 . 6 1}$ & -0.37 & -0.07 \\
\hline Solid colour rate & $\mathbf{0 . 7 4}$ & & \\
\hline
\end{tabular}



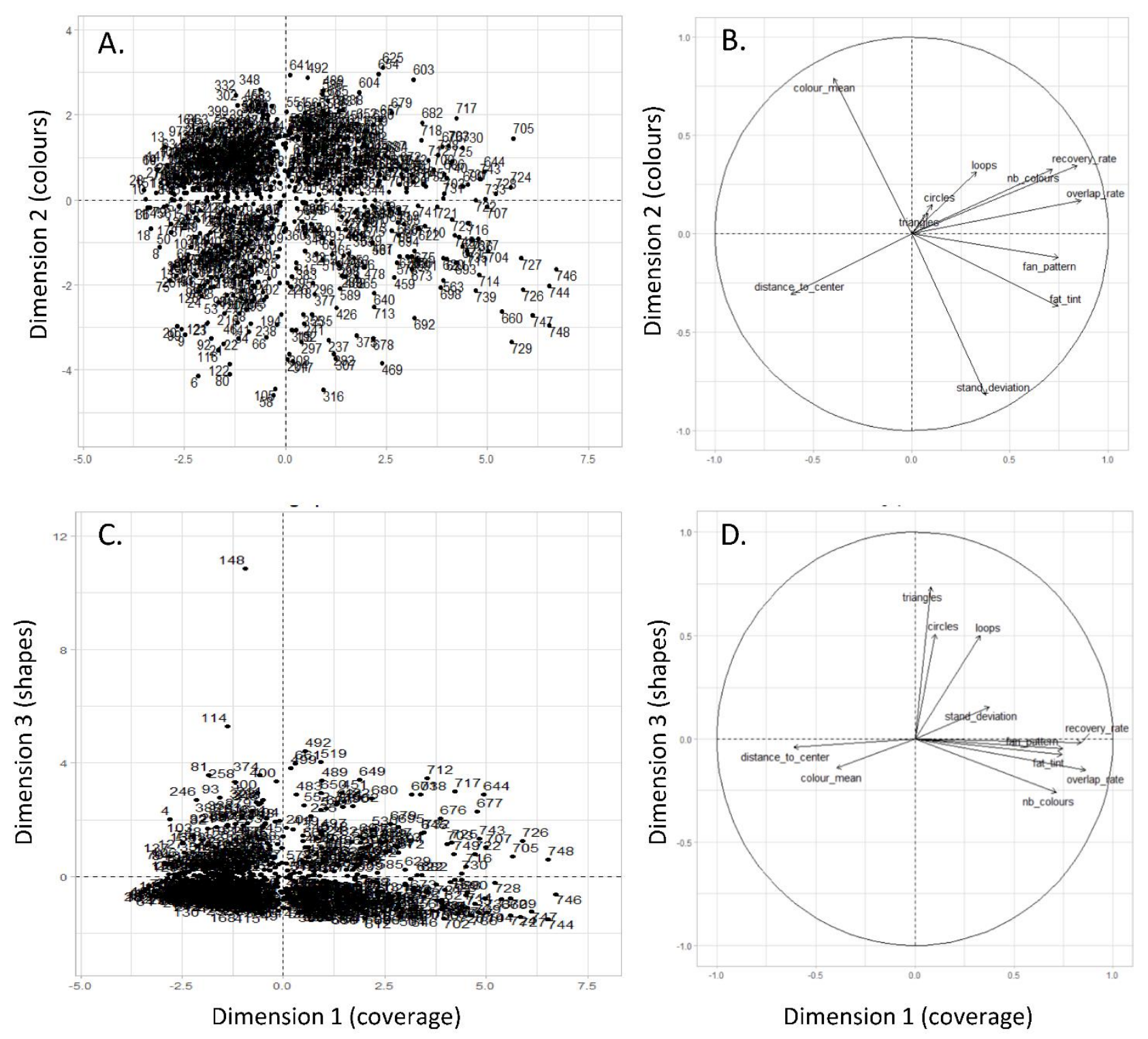

Figure 2: Distribution of data (each point is a drawing) and of variables (each arrow is a variable) respectively for Dimension 1 and Dimension 2 (A., B.) and for Dimension 1 and Dimension 3 (C., D.). The arrow size indicates the correlation with the dimensions.

There were significant differences between the five individuals in the first dimension (Kruskal-Wallis: $\chi^{2}=50.31, \mathrm{p}<0.001$ ). Pairwise comparisons revealed that Molly had higher values than Gypsy and Kiki. Kiki also differed from Julie and Yuki (see pairwise statistics in Table S1, Figure 3a). There were significant differences between orang-utans in the second dimension $\left(\chi^{2}=150.48 \mathrm{p}<0.001\right)$. Pairwise comparisons revealed that Molly had significantly higher values than all other individuals. Julie had lower values than Kiki and Yuki (Table S1, Figure 3b). There were significant differences between individuals in the third dimension $\left(\chi_{2}^{2}=44.51, \mathrm{p}<0.001\right)$ with differences between Gypsy and Molly (Table S1, Figure 3c). Statistical analyses for each of the 12 metrics are given in the Supplementary Material section (Table S2-4, Figure S5). The metrics of Dimension 1 are all different between individuals $\left(\chi_{2}^{2}>11.53, \mathrm{p}<0.02\right)$ with data for Molly being globally different from other individuals (more recovery, more overlap, more colours, closer to the centre) and Kiki showing different results to those of Yuki. Indeed Kiki presented the lowest values for recovering, overlapping and colours whilst showing the 
second highest values after Molly. Although the metrics of the second dimension also revealed interindividual differences $\left(\chi_{2}^{2}>40.08, p<0.001\right)$, the pairwise comparisons yielded more restricted results with only Molly showing very low values and Kiki showing very high values of standard deviation of the colorimetric profile. This indicates that Molly was filling the sheet and showing less contrast, whilst Kiki's drawing showed high contrast due to the few but strong marks on the paper (see Figure 1). Finally, the only difference between individuals in Dimension 3 concerned the number of loops $\left(\chi_{2}^{2}=25.07, p<0.001\right)$, with Molly drawing more loops than Kiki.

a) Dimension 1 : coverage

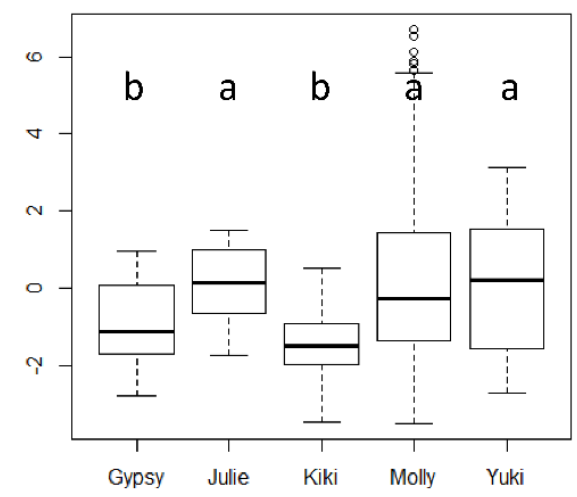

b) Dimension 2 : colours

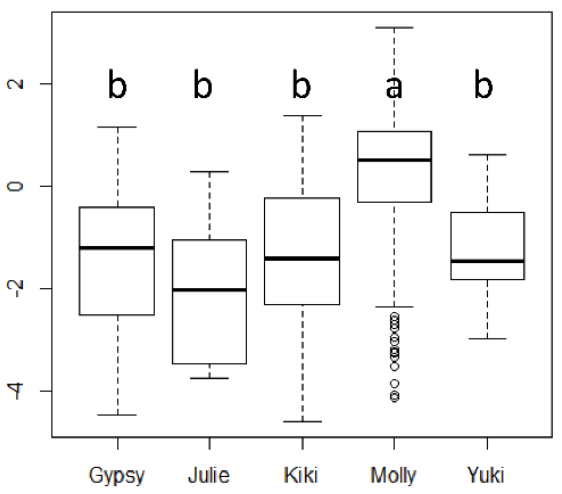

c) Dimension 3 : shapes

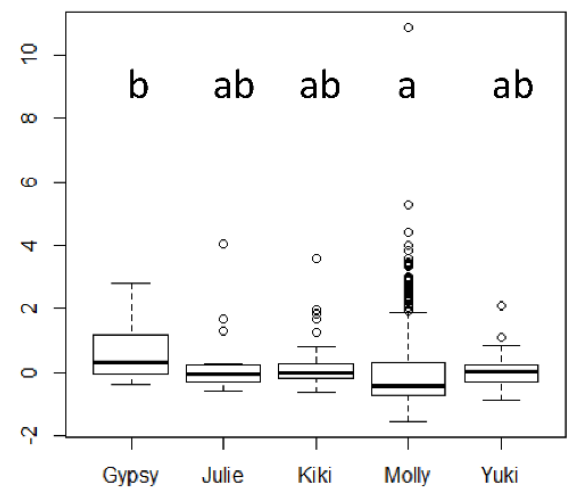

Figure 3: Boxplots showing the differences between individuals in each of the three drawing dimensions. Individuals having different letters show statistical differences.

The main colour found in each drawing revealed a non-random and significant difference in the colours used between individuals (Figure S6). For Kiki $\left(\chi^{2}=62,647, \mathrm{p}\right.$ $<0.001)$ and Molly $\left(\chi^{2}=289.07, \mathrm{p}<0.001\right)$, the main colour in the drawings is green (representing $27.45 \%$ and $22.6 \%$ of the drawings, respectively). For Gypsy $\left(\chi^{2}=41.72, \mathrm{p}\right.$ $<0.001)$, Julie $\left(\chi^{2}=29, \mathrm{p}=0.002\right)$ and Yuki $\left(\chi^{2}=53.2, \mathrm{p}<0.001\right)$, the main colour in the drawings is red (representing $40 \%, 33.3 \%$ and $36.67 \%$ of the drawings, respectively).

\subsection{Longitudinal changes in Molly's drawings}

The number of drawings by Molly over the six years is shown in Figure S7. Three dimensions were retained in the PCA. Their sum represented $64.3 \%$ of the explained variance of the dataset (dimension 1 : eigenvalue $=4.26$, variance $=38.7 \%$; dimension 2 : eigenvalue $=1.60$, variance $=14.5 \%$; dimension 3 : eigenvalue $=1.21$, variance $=11 \%$ ). Metrics are found in the same three dimensions for Molly as for the four other individuals (Table S5). The effect of 3-month periods and seasons on the three dimensions of the PCA were calculated. There was a significant effect of both seasons (linear model with permutation test: $p<0.0001)$ and periods $(p<0.0001)$ on the first dimension. The filling behaviour of drawings decreased with the periods (linear regression: $\mathrm{t}$-value $=-4.7, \mathrm{p}<0.001, \mathrm{R}^{2}=0.03, \mathrm{~F}=22.73$, Figure 4a). Pairwise comparisons revealed that winter had significantly lower values than all other seasons $(p<0.005$, figure $4 \mathrm{~b}$ ). There was no significant effect of the seasons or of the periods on the second $(\mathrm{p}=0.162$ and $\mathrm{p}=0.869$ respectively) or on the third dimension $(\mathrm{p}=0561$ and $\mathrm{p}=0.769$ respectively). More precisely, Molly's drawings differed according to the seasons and time in terms of the coverage rate $(p=0.002$ and $p<0.0001$ respectively), the overlap rate $(p=0.002$ and $p<0.0001)$, the solid colour rate $(p<0.0001$ and $p<0.0001)$, the distance to the centre $(p=0.04$ and $p=0.0002)$ and the fan patterns $(p=0.045$ and $p<0.0001)$, with the winter globally always being different from other seasons. However the number of colours used does not differ according to time $(\mathrm{p}=0.09)$ or the seasons $(\mathrm{p}=0.273)$. Although Dimension 2 did not show differences for the time and the period, the mean 
colorimetric profile showed differences according to the season $(p=0.009)$ with higher values in winter $(p<0.03)$ than other seasons, because Molly filled the paper sheet less in winter, thus making these drawings brighter. Dimension 3 did not show any differences according to time and period, but the number of loops decreased with time $(\mathrm{p}=0.02, \mathrm{z}-$ score $=-2.29)$ and was higher in summer $(\mathrm{p}<0.045)$ compared to other seasons. Other metrics did not show any difference according to time or seasons.

The analysis of the main colour qualitative variable revealed a non-random and a significant difference in the main colour used by Molly in the four seasons (Figure S8): winter $\left(X^{2}=100.16, p<0.001\right)$, spring $\left(X^{2}=90,912, p<0.001\right)$, summer $\left(X^{2}=87,385, p<\right.$ $0.001)$, and autumn $\left(X^{2}=103.27, p<0.001\right)$. Molly preferred green in summer and winter and swapped to pink for spring and autumn. Purple is used more in spring compared to other seasons.

a) Effect of seasons on Molly's drawings

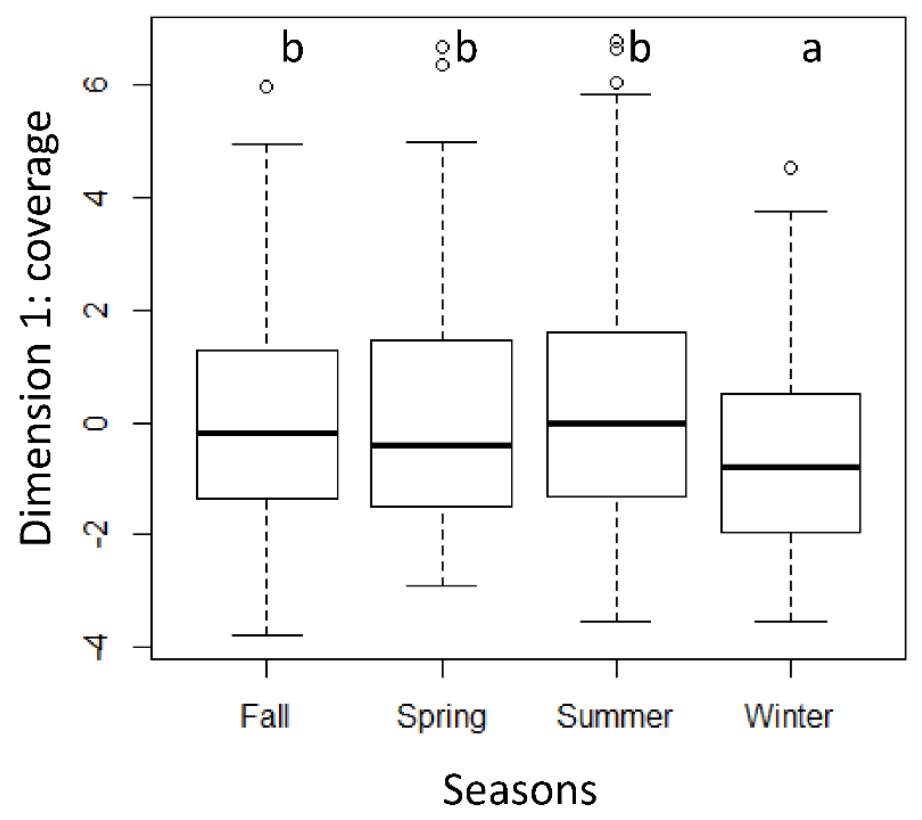

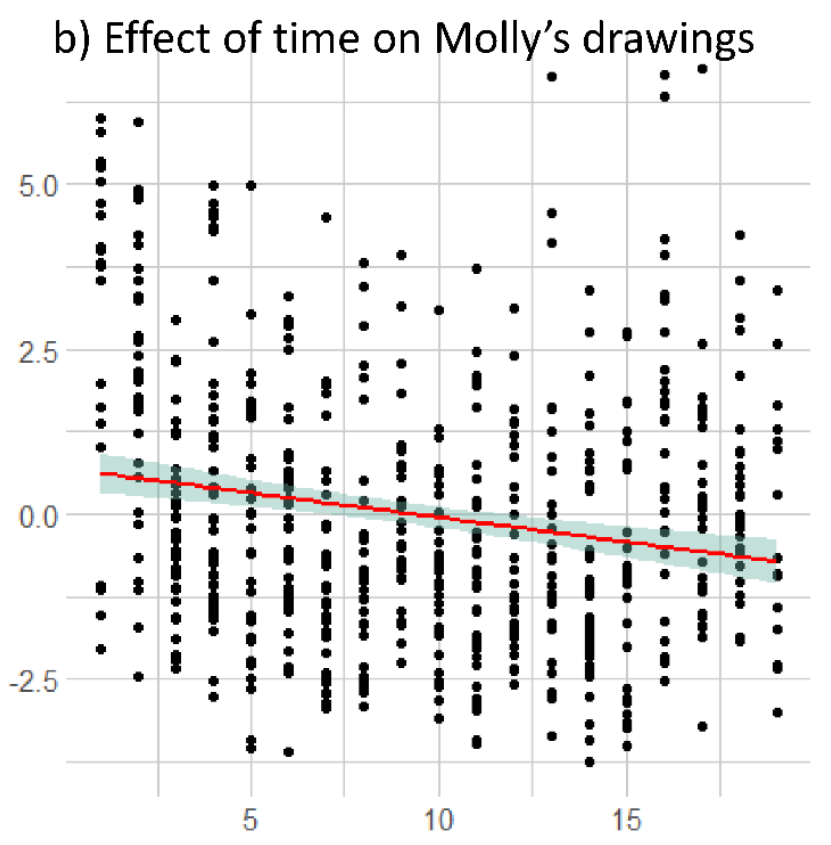

3-month periods

effect of a) seasons and b) time on Dimension 1 (coverage) of Molly's drawings

\section{Discussion}

We studied drawings by orang-utans in order to consider their cognitive capacities phylogenetically within the framework of other hominoids like humans or chimpanzees. In particular, we looked for differences in the drawing behaviour of five female orangutans, one of which was in her last years of life. We found differences between individuals and observed temporal changes in Molly's drawings. The principal component analysis reduced the number of variables to three dimensions that had biological, cognitive meaning, namely the filling aspect, the colour or contrast aspect and the shape aspect.

Most of the time, orang-utans used several colours in their drawings. Zeller [20] found that blue was the most commonly used colour used in the drawings of apes (including 
orang-utans) and children. The main colour used in our orang-utan's drawings differed between individuals. As orang-utans see colours as we do [33], this difference could reflect either an aesthetic or an emotional preference [34]. In children, colours are linked to emotion [34], including in their drawing [21]. This link with emotion may have an evolutionary origin concerning mate choice and competition [35]. Molly, who usually used green and pink colours, mainly chose the colour red when another orang-utan was giving birth [11]. All the individuals drew patterns that were described in Kellogg's children's scribbles classification [23]. Like children, orang-utans can draw multiple lines (called fan patterns), loops and circles. Orang-utans have more dexterity than other great apes since they can draw curved lines, unlike other great apes [20]. Orang-utans have also drawn triangles [36]. Thus, in a way, orang-utans draw better than other non-human apes. The drawing technique used by orang-utans might also be interesting. Previous studies showed that orang-utans often hold the drawing tool between their fingers. However, one female was regularly observed laying the pastel on the sheet before rolling it with her hand [36]. This behaviour could explain some large fan patterns observed in orang-utan drawings. In many studies, primates demonstrated an ability to draw fan patterns. We can cite the chimpanzees Congo and Bella, and the capuchin monkeys Pablo [17]. Drawings by chimpanzees, human children and orang-utans have therefore a lot in common and these new findings may enrich the phylogeny of drawing behaviour among primates.

Our comparative study revealed differences in drawing behaviour among orang-utans, especially for the filling dimension. Here, it is not simply a question of one individual standing out from other individuals, but several differences observed between the five individuals: the drawings by Molly are the most complex (more amply filled than pictures by other individuals, with the use of more shapes and colours) followed by those drawn by Yuki. Kiki also shows differences to the other individuals with her simple but strongly marked drawing (i.e. one colour used, pressing hard on the pencil). We can attribute these differences to personality, motivation or even to different cognitive abilities between individuals. Studies in human children suggest that these interindividual differences might be due to varying levels of cognitive skill maturation [37] and the different speeds at which children learn to draw [2]. According to Willats [38], there may be an interrelation between a child's stage in their drawing skill development and their increasing comprehension of their living world. Our orang-utans have different life experiences. While Molly, who did more complex drawings, was born in the wild and had lived in two zoological parks and given birth 4 times, Kiki, who produced fewer complex drawings, was born in captivity and quickly moved to Tama Zoological Park where she gave birth to one baby. Molly had also lost her sight in one eye between 1993 and 1996 (personal communications from Mr. Kurotori, Tama zookeeper). These different experiences could perhaps explain the differences found in the way they drew. We can also mention the age difference between Molly, who was 54 years old at the beginning of her drawing period, and Kiki who was the youngest of the study group at just 10 years old. Kiki's minimalist use of colours and space in the paper was evidence of either her drawing style or a lack of motivation or interest in the drawing activity. However, Kiki was not the least experienced of the individuals in terms of drawing, even if she was the youngest: she produced 60 drawings whereas 44-year-old orang-utan Julie produced just 16 drawings.

Data for Molly showed a higher mean and a lower standard deviation of the colour spectrum. This indicates a higher diversity in Molly's drawings, many of which are bright, and have lower levels of contrast. Indeed, although Molly did sometimes fill the sheet, particularly with fan patterns - as described for the chimpanzee Congo [17] many of her drawings were almost empty. Other orang-utans showed smaller patterns, which contrasted with the white background. Moreover, Molly seemed to press less on pencils than other individuals, which explains the lower contrast (lowest standard 
deviation of the colour spectrum) in comparison to data for other individuals. The drawings of our orang-utans (and especially those drawn by Molly) confirm the findings of Smith [18], who was the first to report that chimpanzees tended to draw near the centre of the page. Zeller [20] confirmed this finding for other apes. The inter-individual differences we describe in orangutans drawing in terms of the way individuals draw suggest the existence of different personalities, as first suggested by Morris [17]. The wide difference in the number of productions per individual also shows different levels of interest in the activity; this finding confirms previous observations in orang-utans but also in chimpanzees $[12,13,20]$.

The longitudinal study then demonstrated that Molly's drawings evolved over time and between seasons. She used fewer colours, less space and drew further from the centre as the years progressed. At the end of her life, Molly had fat deposits above her eyes and had to use her hands to lift it out of the way, meaning that she could use her hands less to draw. She also became blind in her left eye, which diminished her visual field. Regarding the difference observed in winter, the results tend to show that Molly's frame of mind changed. The orang-utans in our study group do not go outside in winter, and the weather in Japan is very cold for them. They might become bored, and their motivation may decrease. In this way, our results show that Molly does not systematically use the same main colour in her drawings for all seasons: while she preferred purple as main colour in spring (in $23.4 \%$ of her drawings), she used it much less in summer $(7.25 \%)$, autumn (3.27\%) and winter (5.45\%). As Hanazuka and his colleagues [11] hypothesised for non-human primates, drawings could be a window into the internal state of the orang-utan. Moreover, there are no visitors during winter. Evidence that Molly draws more loops and changes the colour she uses in summer could be a cue indicating a good mood due to the weather and the presence of more visitors. Further research is needed to assess whether this number of loops is just the reflect of Molly's motivation or have a more cognitive aspect as symbolism [39].

However, this study is limited by the enrichment origin of these drawings, which restrict the possibilities of controlled conditions. Several drawings were covered with stains and were dirty, with some shapes overlapping. It is difficult to be precise and objective when measuring variables such as the shapes present in the drawing or the predominant colour, especially when water had blurred the patterns. In the future, we could therefore use touch-screen tablets instead of paper [2]. The use of a tablet requires a genuine trust between the researcher and the animal, established from an early age so as not to put the researcher in danger [40]. Drawing on tablets has several advantages such as the extraction of more important information such as temporal data. This method will reveal differences between individuals in terms of order of colours used but also the fractality of the patterns [2]. It would also be interesting to study drawings by males to see if these preferences change according to sex, although this may be difficult as males have previously demonstrated less interest in this task [36]. Another question pertains to the part of the body orang-utans use to draw. Indeed, some apes use their hands while others use their mouth [36].

Overall, the fact that orang-utans draw freely without constraint shows that they are capable of doing so. It would be interesting to study these individuals further and also to study mother-offspring pairs in order to identify a possible transmission of the motivation or ability to draw. Cultural transmission of habits has been shown for example in chimpanzees [41], and in orang-utans [42]. This type of study would contribute to our understanding of the origins of drawings in humans.

\section{Conclusions}


Overall, the fact that orang-utans draw freely without constraint shows that they are capable of doing so. It would be interesting to study these individuals further and also to study mother-offspring pairs in order to identify a possible transmission of the motivation or ability to draw. Cultural transmission of habits has been shown for example in chimpanzees [41], and in orang-utans [42]. This type of study would contribute to our understanding of the origins of drawings in humans.

Supplementary Materials: The following are available online at www.mdpi.com/xxx/s1

Author Contributions: Conceptualization, X.X. and Y.Y.; methodology, X.X.; software, X.X.; validation, C.S. \& M.P.; formal analysis, G.T., A.L., M.P. \& C.S.; investigation, G.T., A.L., M.P. \& C.S.; resources, N.E. \& S.M.; data curation, N.E. \& S.M.; writing-original draft preparation, C.S \& G.T.; writing-review and editing, all authors; visualization, C.S, A.L. \& G.T.; supervision, C.S., M.P. \& M.S.; project administration, C.S., M.P. \& M.S.; funding acquisition, C.S., M.P. \& M.S.. All authors have read and agreed to the published version of the manuscript.

Funding: This project has received financial support from the CNRS through the MITI interdisciplinary programs, the University of Strasbourg through an IDEX Exploratory Research program and from a KAKENHI program.

Institutional Review Board Statement: The Tama Zoological Park Ethics Board approved this noninvasive behavioural study, which complied with the Code of Ethics for Japanese Association of Zoos and Aquariums.

Data Availability Statement: The data presented in this study are available on request from the corresponding author. The data are not publicly available due to Tama zoo request and copyright.

Acknowledgments: We are grateful to the Tama Zoological Park in Japan for the providence of orang-utans drawings.

Conflicts of Interest: The authors declare no conflict of interest. The funders had no role in the design of the study; in the collection, analyses, or interpretation of data; in the writing of the manuscript, or in the decision to publish the results.

\section{References}

1. Martinet, L.; Pelé, M. Drawing in Nonhuman Primates: What We Know and What Remains to Be Investigated. Journal of comparative psychology (Washington, D.C. : 1983) 2020.

2. Martinet, L.; Sueur, C.; Hirata, S.; Hosselet, J.; Matsuzawa, T.; Pelé, M. New Indices to Characterize Drawing Behavior in Humans (Homo Sapiens) and Chimpanzees (Pan Troglodytes). Sci Rep 2021, 11, 3860.

3. Locke, D.P.; Hillier, L.W.; Warren, W.C.; Worley, K.C.; Nazareth, L.V.; Muzny, D.M.; Yang, S.-P.; Wang, Z.; Chinwalla, A.T.; Minx, P.; et al. Comparative and Demographic Analysis of Orang-Utan Genomes. Nature 2011, 469, 529-533, doi:10.1038/nature09687.

4. Damerius, L.A.; Burkart, J.M.; Van Noordwijk, M.A.; Haun, D.B.M.; Kosonen, Z.K.; Galdikas, B.M.F.; Saraswati, Y.; Kurniawan, D.; Van Schaik, C.P. General Cognitive Abilities in Orangutans (Pongo Abelii and Pongo Pygmaeus). Intelligence $2019,3-11$.

5. Dufour, V.; Pelé, M.; Neumann, M.; Thierry, B.; Call, J. Calculated Reciprocity after All: Computation behind Token Transfers in Orang-Utans. Biology Letters 2009, 5, 172-175, doi:10.1098/rsbl.2008.0644.

6. Lethmate, J. Tool-Using Skills of Orang-Utans. Journal of Human Evolution 1982, 11, $49-64$.

7. Bardo, A.; Cornette, R.; Borel, A.; Pouydebat, E. Manual Function and Performance in Humans, Gorillas, and Orangutans during the Same Tool Use Task. American Journal of Physical Anthropology 2017, 164, 821-836, doi:10.1002/ajpa.23323.

8. Mendes, N.; Steinbeis, N.; Bueno-Guerra, N.; Call, J.; Singer, T. Preschool Children and Chimpanzees Incur Costs to Watch Punishment of Antisocial Others. Nature human behaviour 2018, 2, 45-51.

9. Damerius, L.A.; Burkart, J.M.; van Noordwijk, M.A.; Haun, D.B.; Kosonen, Z.K.; Galdikas, B.M.; Saraswati, Y.; Kurniawan, D.; van Schaik, C.P. General Cognitive Abilities in Orangutans (Pongo Abelii and Pongo Pygmaeus). Intelligence 2019, 74, 3-11. 
10. Mackinnon, J. The Behaviour and Ecology of Wild Orang-Utans (Pongo Pygmaeus). Animal Behaviour 1974, $22,3-74$.

11. Hanazuka, Y.; Kurotori, H.; Shimizu, M.; Midorikawa, A. The Effects of the Environment on the Drawings of an Extraordinarily Productive Orangutan (Pongo Pygmaeus) Artist. Frontiers in Psychology 2019, 10, 2050.

12. Boysen, S.T.; Berntson, G.G.; Prentice, J. Simian Scribbles: A Reappraisal of Drawing in the Chimpanzee (Pan Troglodytes). J Comp Psychol 1987, 101, 82-89.

13. Tanaka, M.; Tomonaga, M.; Matsuzawa, T. Finger Drawing by Infant Chimpanzees (Pan Troglodytes). Anim Cogn 2003, 6, 245251.

14. DeLoache, J.S.; Pickard, M.B.; LoBue, V. How Very Young Children Think about Animals. 2011.

15. Casti, A.B. Reaction to Stimulus Figures in Chimpanzee Drawings. Master thesis, Central Washington University: USA, 2016.

16. Schiller, P.H. Figural Preferences in the Drawings of a Chimpanzee. Journal of Comparative and Physiological Psychology 1951, 44, 101.

17. Morris, D. The Biology of Art: A Study of the Picture-Making Behaviour of the Great Apes and Its Relationship to Human Art; Taylor \& Francis, 1962;

18. Smith, D.A. Systematic Study of Chimpanzee Drawing. J Comp Physiol Psychol 1973, 82, 406-414.

19. Adi-Japha, E.; Levin, I.; Solomon, S. Emergence of Representation in Drawing: The Relation between Kinematic and Referential Aspects. Cognitive Development 1998, 13, 25-51.

20. Zeller, A. 'What's in a Picture?' A Comparison of Drawings by Apes and Children. Semiotica 2007, 2007, $181-214$.

21. Crawford, E.; Gross, J.; Patterson, T.; Hayne, H. Does Children's Colour Use Reflect the Emotional Content of Their Drawings? Infant and Child Development 2012, 21, 198-215.

22. Sueur, C.; Pelé, M. Importance of living environment for the welfare of captive animals: behaviours and enrichment. In Animal Welfare: From Science to Law; 2019; pp. 175-188.

23. Kellogg, R. Analyzing Children's Art; National Press Books: Palo Alto, Calif., 1969;

24. Peterson, B.G.; Carl, P. PerformanceAnalytics: Econometric Tools for Performance and Risk Analysis; 2020;

25. Lê, S.; Josse, J.; Husson, F. FactoMineR: A Package for Multivariate Analysis. Journal of Statistical Software 2008, 25, 1-18.

26. Kaiser, H.F. The Varimax Criterion for Analytic Rotation in Factor Analysis. Psychometrika 1958, 23, 187-200.

27. Hothorn, T.; Hornik, K.; Wiel, M.A. van de; Zeileis, A. Implementing a Class of Permutation Tests: The Coin Package. Journal of Statistical Software 2008, 28, 1-23.

28. R Core Team R: A Language and Environment for Statistical Computing; R Foundation for Statistical Computing: Vienna, Austria, 2020;

29. Fox, J.; Weisberg, S. An R Companion to Applied Regression; Third.; Sage: Thousand Oaks CA, 2019;

30. Giraudoux, P. Pgirmess: Spatial Analysis and Data Mining for Field Ecologists. R Package Version 1.6.9. 2018.

31. Good, P. Permutation, Parametric and Bootstrap Tests of Hypotheses. 3rd Ed. 2005.

32. Mangiafico, S. Rcompanion: Functions to Support Extension Education Program Evaluation; 2021;

33. Tigges, J. On Color Vision in Gibbon and Orang-Utan. Folia Primatologica 1963, 188-198.

34. Mikellides, B. Colour psychology: The emotional effects of colour perception. In Colour Design; Elsevier, 2012; pp. 105-128.

35. Rowland, H.M.; Burriss, R.P. Human Colour in Mate Choice and Competition. Philosophical Transactions of the Royal Society B: Biological Sciences 2017, 372, 20160350.

36. Vancatova, M. Creativity and innovative behaviour in primates on the example of picture-making activity of apes. Creativity and innovative behaviour in primates on the example of picture-making activity of apes 2008.

37. Saito, A.; Hayashi, M.; Takeshita, H.; Matsuzawa, T. The Origin of Representational Drawing: A Comparison of Human Children and Chimpanzees. Child Development 2014, 85, 2232-2246.

38. Willats, J. Making Sense of Children's Drawings; Psychology Press, 2006; ISBN 978-1-135-62498-9.

39. Gardner, H.; Wolf, D. The Symbolic Products of Early Childhood. 1987. 
40. Matsuzawa, T. The 40th Anniversary of the Ai Project: The Commemorative Gift Is a Silk Scarf Painted by Ai the Chimpanzee. Primates 2017, 58, 261-265.

41. Whiten, A.; Goodall, J.; McGrew, W.C.; Nishida, T.; Reynolds, V.; Sugiyama, Y.; Tutin, C.E.; Wrangham, R.W.; Boesch, C. Cultures in Chimpanzees. Nature 1999, 399, 682-685, doi:10.1038/21415.

42. Schaik, C.P. van; Ancrenaz, M.; Borgen, G.; Galdikas, B.; Knott, C.D.; Singleton, I.; Suzuki, A.; Utami, S.S.; Merrill, M. Orangutan Cultures and the Evolution of Material Culture. Science 2003, 299, 102-105. 